\title{
Les urgences médicales dans les pôles et services d'odontologie des centres hospitaliers universitaires français
}

\section{Medical emergencies in university dental teaching centers of french hospitals}

\author{
FLORIAN LAURENT ${ }^{1}$, PASCAL AUGUSTIN ${ }^{3}$, NICOLAS SEGAL ${ }^{4}$, CATHY NABET ${ }^{2}$, PHILIPPE LESCLOUS ${ }^{1}$, LOUIS MAMAN
}

\begin{abstract}
RÉSUMÉ
Objectif : Cette enquête a pour but de recenser les urgences médicales survenant dans les services d'odontologie et de faire l'état des moyens, de la formation et des protocoles mis en œuvre pour y faire face.

Méthode : Elle a été menée de décembre 2007 à juin 2008 auprès des pôles et services d'odontologie des centres hospitaliers universitaires français. Un questionnaire portant sur le matériel d'urgence disponible, la formation des praticiens, les procédures d'urgence et la survenue des urgences médicales sur une période de un an a été envoyé à tous les chefs de services.

Résultats : Sur les 32 pôles et services contactés, 28 ont répondu au questionnaire. Le matériel d'urgence disponible est très variable selon les services. La majorité d'entre eux ne dispose pas des médicaments recommandés ni du matériel spécialisé nécessaire tel qu'un défibrillateur. Concernant la formation des praticiens, $75 \%$ d'entre eux n'ont eu aucune formation particulière pour la gestion des urgences médicales. Dans près de la moitié des services, il n'y a pas de procédure définie pour faire appel à des moyens de secours extérieurs au service. On relève une moyenne de 20 urgences médicales par an et par service. La majorité d'entre elles sont des malaises vagaux mais on retrouve également des accidents plus graves qui peuvent mettre en jeu le pronostic vital du patient.

Conclusion : Même si l'incidence des urgences médicales semble faible dans les services hospitaliers d'odontologie, il est nécessaire que les praticiens disposent du matériel et soient formés à leur prise en charge. (Med Buccale Chir Buccale 2009 ; 15: 87-92).
\end{abstract}

mots clés: urgence médicale, service d'odontologie, défibrillateur, dentiste, AFGSU

\section{SUMMARY}

Objective : The aim of this study was to audit medical emergencies occurring in dental teaching centers and to describe equipments and protocols for their management. Indeed, we wanted to describe the formation of caregivers in the area of medical emergencies.

Methods: This study was carried out from december 2007 to june 2008 in dental teaching centers of French hospitals. A questionnaire was sent to the chief of each department. The questionnaire concerned the available equipment, caregivers formation, medical protocols and the medical emergencies that occurred during one year.

\begin{tabular}{l}
$\begin{array}{l}\text { médecine } \\
\text { buccale } \\
\text { chirurgie } \\
\text { buccale }\end{array}$ \\
\hline VOL. 15, N ${ }^{\circ} 2$ \\
2009 \\
\hline page 87
\end{tabular}

1. Département de Médecine Buccale et de Chirurgie Buccale Université Paris 5 (Paris Descartes)
2. Département de santé publique Université Paris 5 (Paris Descartes)
3. Département d'Anesthésie Réanimation Groupe hospitalier Bichat Claude Bernard Université Paris 7 (Denis Diderot)
4. Département d'Anesthésie Réanimation Groupe hospitalier Lariboisière Université Paris 7 (Denis Diderot)

Demande de tirés à part:

Florian Laurent Faculté de Chirurgie Dentaire Département de Médecine Buccale et de Chirurgie Buccale 1 rue Maurice Arnoux 92120 Montrouge florian.laurent@free.fr 
Results : Beyond the 32 dental centers, 28 answered the questionnaire. The analysis revealed a marked heterogeneity in the available equipment for management of medical emergencies. Most of the centers didn't possess the recommended drugs or needed equipment, including defibrillators. Seventy five percents of health professionals didn't have any training in the management of medical emergencies. Nearly half of the centers hadn't defined any guideline for the alert of emergency service. A mean of 20 medical emergencies per year per center were declared. Most of them were benign vasovagal episodes, but some life-threatening emergencies were also reported. Conclusion: The medical emergencies incidence seems to be low in the dental teaching centers. Nevertheless, each center should possess appropriate training, equipments and procedures to manage it adequately. (Med Buccale Chir Buccale 2009 ; 15: 87-92).

key words: medical emergencies, dental teaching center, defibrillator, dentist

médecine

buccale

chirurgie

buccale

VOL. $15, \mathrm{~N}^{\circ} 2$ 2009

page 88
La pratique de l'Odontologie présente des risques de complications générales, notamment au travers des gestes invasifs pratiqués sur les patients (anesthésies, interventions chirurgicales, etc.). Les produits et les médicaments utilisés quotidiennement peuvent également interagir avec l'état général, le traitement ou une sensibilité particulière du patient. Enfin, les praticiens peuvent être confrontés à une urgence inopinée ou à la décompensation d'une affection du patient.

Par ailleurs, les patients qui fréquentent les services hospitaliers d'odontologie sont généralement à risque du fait des maladies qu'ils présentent ou des traitements qu'ils suivent.

Plusieurs études sur les urgences médicales dans les cabinets dentaires libéraux français ont été publiées mais, à notre connaissance, il n'existe pas de données concernant les services hospitaliers d'odontologie. C'est pourquoi de décembre 2007 à juin 2008, une enquête a été menée auprès de ces services.

Cette étude a pour but de faire l'état des moyens, de la formation et des protocoles mis en œuvre dans ces services pour répondre à une situation médicale d'urgence. Elle a également pour objectif de recenser les différents types d'urgence rencontrée et leur fréquence.

\section{MATÉRIEL ET MÉTHODE}

En décembre 2007, un questionnaire sur les urgences médicales a été adressé à tous les responsables de pôles et de services d'odontologie des centres hospitaliers universitaires français.
Les réponses ont été collectées jusqu'en juin 2008.

Ce questionnaire se divise en quatre parties et aborde successivement :

- le matériel d'urgence disponible dans le service (matériel de diagnostic, d'injection et de perfusion, de réanimation et médicaments d'urgence) - la formation des praticiens dans le domaine des urgences médicales

- les procédures d'urgence

- la survenue des situations d'urgence durant une période d'un an, entre le $1^{\text {er }}$ octobre 2006 et le $1^{\text {er }}$ octobre 2007.

Concernant le matériel nécessaire pour traiter une urgence médicale dans un service hospitalier, la Société Française d'Anesthésie et de Réanimation (SFAR) a publié en 2004 des recommandations [5] qui décrivent notamment la composition minimum du chariot d'urgence qui doit se trouver dans chaque service hospitalier (Annexe 1). Dans cette étude, nous comparerons l'équipement des services d'odontologie avec ces recommandations.

\section{RÉSULTATS}

Sur les 32 pôles et services contactés, 28 ont répondu au questionnaire. Certaines informations ont été difficiles à obtenir ; sur les 28 questionnaires, seulement 9 ont été remplis complètement. Les 19 autres n'ont pas pu être exploités dans leur intégralité. Les paramètres qui manquent concernent principalement la formation et l'épidémiologie. Trois rubriques n'ont pu être 


\section{Annexe 1 \\ DOTATION MINIMALE DU CHARIOT D'URGENCE [5]}

- Défibrillateur semi-automatique (DSA), si possible débrayable en mode manuel ;

- Bouteille d'oxygène à manodétendeur intégré, vérifiée et prête à l'emploi ;

- Médicaments : adrénaline, dérivés nitrés (injectable et spray), atropine, lidocaïne, amiodarone, furosémide, soluté glucosé à $30 \%$, benzodiazépine injectable, bêta2-adrénergiques (spray et solution pour aérosol), solutés de perfusion conditionnés en poche souple ( $\mathrm{NaCl}$ à 0,9 \%, colloïdes de synthèse) ;

- Matériel de ventilation : canules de Guedel $n^{\circ} 2$ et 3 , masques faciaux $n^{\circ} 3$ à 6 , ballon autoremplisseur à valve unidirectionnelle et filtre antibactérien, masques pour aérosol, sondes à oxygène, tuyaux de connexion, masques à haute concentration ;

- Matériel pour accès veineux et injections : seringues de 5 et de $10 \mathrm{ml}$, jeu d'aiguilles, cathéters courts 14-16-18-20-22 G, perfuseurs avec robinet à 3 voies, compresses stériles, solution anti-septique, garrot, champs adhésifs transparents, adhésif de fixation, gants non stériles, container à aiguilles, lunettes de protection, solution hydro-alcoolique ;

- Matériel d'aspiration : système d'aspiration vérifié et prêt à l'emploi, sondes d'aspiration trachéo-bronchique, sondes pour aspiration gastrique, seringue de Guyon (50 ml à embout conique), sac à urine non stérile, raccords biconiques ;

- Plan dur pour massage cardiaque.

exploitées par manque de données : la formation du personnel infirmier, le mode de prise en charge des urgences médicales et le nombre de passage/patient annuel.

\section{MATÉRIEL D'URGENCE}

\section{Matériel de diagnostic}

Tous les services disposent du matériel nécessaire pour mesurer la pression artérielle et $60 \%$ d'un lecteur de glycémie capillaire. Peu sont équipés de matériel plus spécifique tel qu'un oxymètre de pouls (39\%) ou un moniteur multiparamétrique (7\%).

Huit services (29\%) ont uniquement le matériel pour mesurer la pression artérielle (Fig. 1).

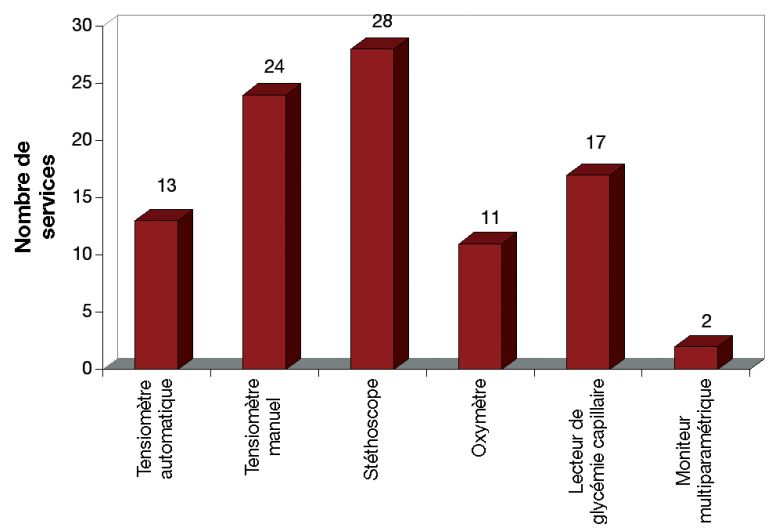

Figure 1 : Matériel de diagnostic disponible dans les services d'odontologie.

Diagnostic equipment in dental teaching centers.

\section{Médicaments}

Seulement deux services détiennent les médicaments recommandés par la SFAR (Annexe 1). On remarque que trois services n'ont pas d'adrénaline (ni en kit, ni en ampoule), quatre n'ont ni glucose ni glucagon, et onze n'ont pas de B2mimétiques (injectables ou en aérosols). Par contre certains services disposent de médicaments tels que : Hypnovel ${ }^{\circledR}$, Heptamyl ${ }^{\circledR}$, Diprivan $^{\circledR}$, Propofol $^{\circledR}$, Anexate $^{\circledR}$, Narcan $^{\circledR}$, Tranxène ${ }^{\circledR}$ (Fig. 2).

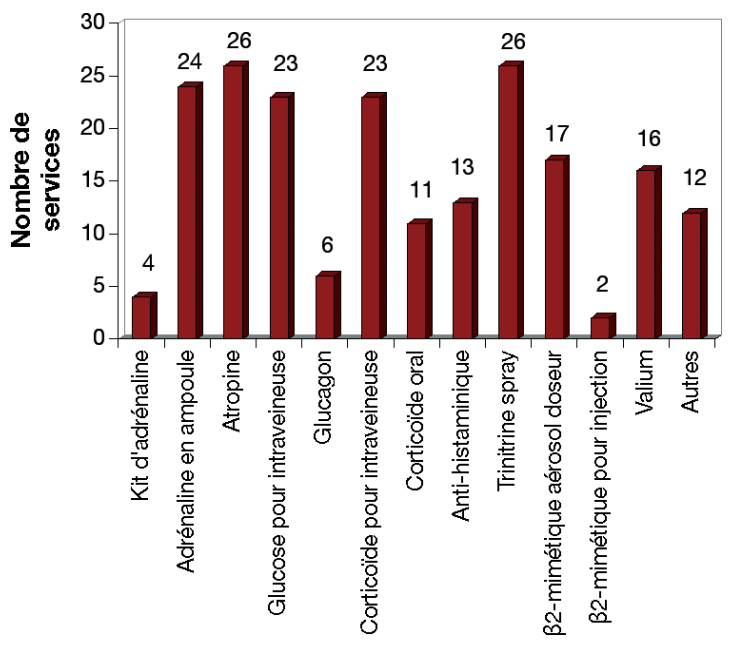

Figure 2 : Médicaments d'urgence disponibles dans les services d'odontologie.

Emergency drugs in dental teaching centers. médecine

buccale chirurgie buccale

VOL. $15, \mathrm{~N}^{\circ} 2$ 2009

page 89 


\section{Matériel d'injection et de perfusion}

Dix-huit services (64\%) disposent du matériel pour réaliser tous les types d'injection (SC, IM, et IV) et vingt-quatre services ont le nécessaire pour poser une voie veineuse périphérique. Certains services possèdent des solutés autres que le sérum physiologique et les sérum glucosés, comme : Voluven ${ }^{\circledR}$, Bicarbonate, Gélofusine ${ }^{\circledR}$, Heafusine $^{\circledR}$, Plasmion $^{\circledR}$, Ringer lactate ${ }^{\circledR}$.

\section{Matériel de réanimation}

On note que $93 \%$ des services disposent d'une bouteille d'oxygène et $14 \%$ détiennent un défibrillateur (automatique ou semi-automatique). Quatre services disposent de matériel d'intubation et un service possède une planche pour massage cardiaque (Fig. 3).

médecine

buccale

chirurgie

buccale

VOL. $15, \mathrm{~N}^{\circ} 2$ 2009

page 90

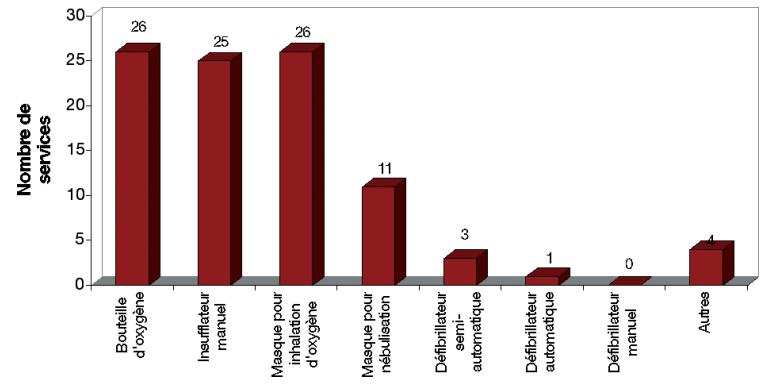

Figure 3 : Matériel de réanimation disponible dans les services.

Emergency equipment in the centers.

\section{Chariot d'urgence}

On remarque que $61 \%$ des services disposent d'un chariot d'urgence et que $25 \%$ regroupent leurs matériels d'urgence sous une autre forme (valise, caisse, mallette ou sac). Les autres services (14 \%) ne regroupent pas leur matériel.

\section{FORMATION DU PERSONNEL}

\section{Praticiens}

A partir des questionnaires, on dénombre 908 praticiens qui encadrent les étudiants. Onze services n'ont pas fourni de renseignements sur la formation de leurs praticiens.
Dans les 17 services qui ont complété la partie formation, on recense 564 praticiens. Soixantequinze pour cent de ces praticiens n'ont reçu aucune formation spécifique dans le domaine des urgences médicales, $7 \%$ possèdent l'Attestation de Formation aux gestes et Soins d'Urgence (AFGSU) et $11 \%$ un diplôme de premiers secours (Attestation de Formation aux Premiers Secours ou Prévention et Secours Civiques de niveau 1). On note que $5 \%$ des praticiens ont suivi une formation dans ce domaine, que ce soit lors de journées organisées par un organisme de formation continue, le service formation de l'hôpital ou le SAMU dont dépend le service (Fig. 4).

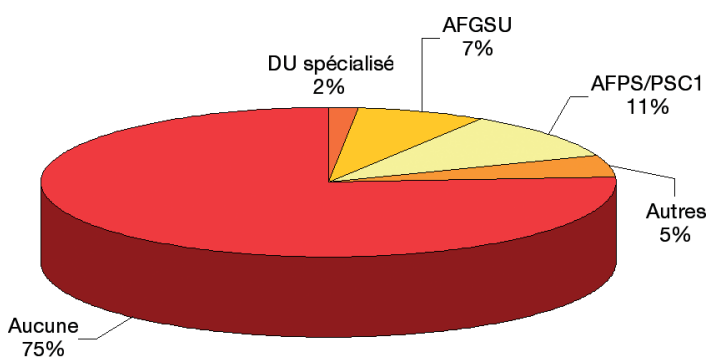

Figure 4 : Formation des praticiens pour traiter les urgences médicales.

Training of the teachers in medical emergencies.

\section{Personnel infirmier}

Vingt-trois services bénéficient de la présence de personnel infirmier durant la totalité ou une partie de leurs plages d'activité (Fig. 5).

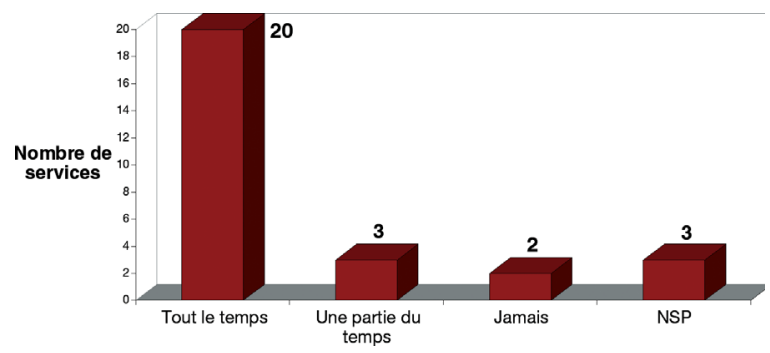

Figure 5 : Présence de personnel infirmier durant la période d'activité du service. (NSP : Ne se prononce pas) Presence of nurse in the service. 


\section{PROCÉDURES D'URGENCE}

\section{Procédures d'urgences}

Trois services n'ont aucune procédure interne définie pour la prise en charge d'une urgence médicale. Dans $54 \%$ des services, c'est l'infirmière qui est appelée dans ce type de situation alors que, dans $46 \%$ des services, c'est le premier enseignant présent qui prend en charge l'urgence. Dans les autres procédures, on retrouve l'appel direct au SAMU - Centre 15 ou d'un médecin réanimateur référent.

Dans $43 \%$ des services, il n'y a pas de procédure définie dans l'hôpital. Pour les autres services, certains font appel à l'interne de garde (54\%), au service des urgences ou de réanimation de l'hôpital (39 \%). Les autres procédures sont l'appel du SAMU ou d'un numéro spécialement prévu pour les urgences (Fig. 6).

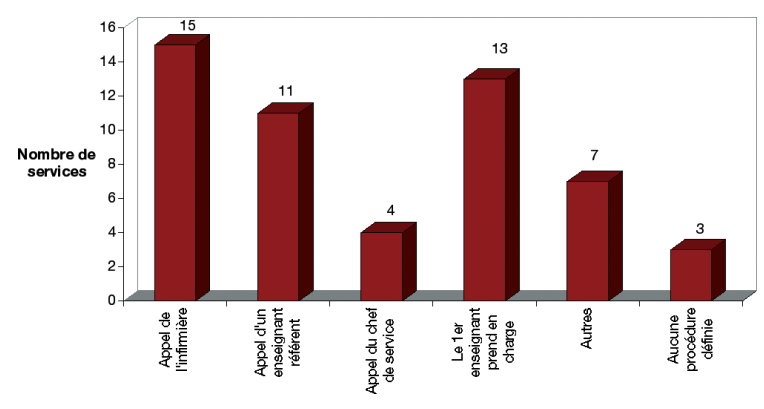

Figure 6 : Procédure d'urgence dans les services. Medical emergencies protocols in the centers.

\section{Protocoles écrits pour les médica- ments d'urgence}

On remarque que $82 \%$ des services n'ont pas de protocoles écrits concernant l'utilisation des médicaments d'urgence. Quatre services ont à disposition des protocoles pour tous les médicaments tandis qu'un service en dispose pour certains médicaments uniquement.

\section{SURVENUE DES URGENCES MÉDICALES}

Vingt trois services (82\%) déclarent avoir été confrontés à des urgences médicales durant la période du $1^{\text {er }}$ octobre 2006 au $1^{\text {er }}$ octobre 2007.
Deux déclarent ne pas y avoir été confrontés et trois ne se prononcent pas.

Sur les 28 questionnaires, 14 ne sont pas exploitables pour fournir des données épidémiologiques (données incomplètes ou non-complétées). Dans les 14 questionnaires exploitables, on recense 282 urgences médicales. Dans ces 14 services, 2 déclarent ne pas en avoir eu durant cette période. En ne tenant compte que de ces 14 services, on obtient une moyenne de 20 urgences par service et par an (Tab. 1).

Tableau 1: Urgences médicales survenue dans les services d'odontologie entre le $1^{\text {er }}$ octobre 2006 et le 1 er octobre 2007.

Medical emergencies in university dental teaching centers from the 1 st of october 2006 to the 1st of october 2007.

\begin{tabular}{|lcc|}
\hline Type d'urgence & Nb & $\%$ \\
\hline Malaises vagaux & 125 & $44,3 \%$ \\
\hline Hyperventilations psychogènes & 67 & $23,8 \%$ \\
\hline Hypoglycémies & 54 & $19,1 \%$ \\
Convulsions & 10 & $3,5 \%$ \\
Crises hypertensives & 6 & $2,1 \%$ \\
Allergies mineures & 5 & $1,8 \%$ \\
Inhalations de corps étrangers & 4 & $1,4 \%$ \\
Ingestions de corps étrangers & 4 & $1,4 \%$ \\
Accidents vasculaires cérébraux & 2 & $0,7 \%$ \\
Syndromes coronariens aigus & 2 & $0,7 \%$ \\
\hline Traumatismes & 2 & $0,7 \%$ \\
\hline Détresse respiratoire aiguë & 1 & $0,4 \%$ \\
\hline
\end{tabular}

\section{DISCUSSION}

Si on compare l'équipement des services hospitaliers d'odontologie avec les recommandations de la Société Française d'Anesthésie et de Réanimation, on constate que la plupart d'entres eux ne disposent pas de l'ensemble du matériel d'urgence nécessaire, notamment d'un défibrillateur. On note également que certains services sont clairement sous-équipés en médicaments d'urgence tandis que d'autres détiennent des médicaments de réanimation non recommandés pour ce type de service. médecine

buccale

chirurgie

buccale

VOL. $15, \mathrm{~N}^{\circ} 2$ 2009

page 91 
médecine buccale chirurgie buccale

VOL. $15, \mathrm{~N}^{\circ} 2$ 2009

page 92
Concernant la formation des praticiens, peu d'entre eux ont bénéficié d'une préparation spécifique pour la gestion des urgences médicales. Pour une partie des praticiens formés, il a s'agit d'une formation de secourisme (Attestation de Formation aux Premiers Secours ou Prévention Secours Civique de Niveau 1) peu adaptée à une prise en charge médicale des urgences. L'Attestation de Formation aux Gestes et Soins d'Urgence de niveau 2 permet de préparer les chirurgiens-dentistes à la gestion des situations d'urgence médicale et aux risques sanitaires qui peuvent survenir dans leur pratique quotidienne. Dans ce contexte où une majorité de praticiens n'est pas formée spécifiquement aux urgences médicales, on remarque que dans près de 9 services sur 10 , ce type de situation est prise en charge par le premier praticien présent. Par ailleurs, peu de services mettent à disposition des protocoles pour l'utilisation des médicaments ou le déclenchement de moyens extérieurs au service. Même si la formation des praticiens est une étape fondamentale de la préparation du service aux urgences médicales, il semble important que ces derniers disposent de matériels et de médicaments adaptés et de protocoles sur l'utilisation des médicaments d'urgence, sur l'alerte de personnes compétentes identifiées au sein du service et sur les possibilités de recours à des moyens extérieurs au service qu'il soient intra- ou extra-hospitaliers.

Enfin, le nombre total d'urgences relevé dans cette étude est sous-estimé par rapport à la réa- lité. En effet, seulement la moitié des services ont renvoyé des données exploitables sur ce sujet. Néanmoins, on peut retenir une moyenne de 20 urgences médicales par an et par service. Les urgences rencontrées sont sensiblement de même type que dans le secteur libéral, avec une majorité de malaises vagaux, d'hyperventilations psychogènes et d'hypoglycémies $[1,2,3,4]$. Ces trois situations représentent à elles seules quasiment 9 urgences médicales sur 10 dans les services hospitaliers d'odontologie. On peut noter l'absence de réaction allergique grave (choc anaphylactique ou œdème de Quincke), de crise d'asthme et d'arrêt cardio-respiratoire. Toutefois, avec une fréquence plus faible, on retrouve des situations pouvant engager le pronostic vital du patient : syndrome coronarien aigu, accident vasculaire cérébral, convulsion, inhalation de corps étranger, etc.

Ainsi, même si l'incidence des urgences médicales dans les services d'odontologie semble faible, leur survenue n'est pas anecdotique. De plus, si la majorité de ces situations peuvent paraître bénignes, certaines peuvent engager le pronostic vital des patients. II parait important que ces services mettent en place une politique de formation des praticiens notamment au travers de l'AFGSU, uniformisent l'équipement en médicaments et en matériels d'urgence en fonction des recommandations de la SFAR et mettent en place des protocoles qui organisent la prise en charge de ces situations pour accroître la sécurité des patients.

\section{RÉFÉRENCES}

1 - Calon B, Bildstein A, Samin J, Meyer A, Attard O, Rieger J, Boukari A, Féki A. Urgences médicales au cabinet dentaire : une enquête française. Med Buc Chir Buc $2007 ; 13: 31-5$.

2 - Dreyphus PH, Foissac JC, Freysz M. Caractéristiques des appels d'urgence au centre 15 provenant des cabinets dentaires. Med Buc Chir Buc 1999; $5:$ 40-1.

3 - Fleuranceau X, Louville Y, Lesclous P. Les urgences médicales au cabinet dentaire : quel bilan ? Info Dent $2004 ; 16$ : 993-1000
4 - Verner C, Louville Y. Les appels aux urgences. Real Clin $1998 ; 9 ; 59-61$.

5 - Recommandations pour l'organisation de la prise en charge des urgences vitales intrahospitalières. Conférence d'experts de la Société Française d'Anesthésie et de Réanimation (SFAR), du Samu de France, de la Société Francophone de Médecine d'Urgence (SFMU) et de la Société de Réanimation de Langue Française (SRLF) 2004. www.sfmu.org 